\title{
LA EDUCACIÓN SOCIAL Y LA VULNERABILIDAD DE LOS NIÑOS QUE PERMANECEN EN LAS CALLES
}

\begin{abstract}
Resumen
A partir del desarrollo del estado de bienestar en Europa -tras la Segunda Guerra Mundial-, en las sociedades occidentales constantemente se han ido ampliando y diversificando las prestaciones y atenciones sociales, así como los escenarios y áreas de la pedagogía social, dirigidas hacia distintos sectores de la población dentro del marco de actuación socioeducativa. En Iberoamérica y concretamente en Perú, la pedagogía social desde el punto de vista académico no tiene una presencia significativa como ocurre en Europa; sin embargo, existe una praxis política y social en diversos ámbitos que se desarrolla tanto desde instancias gubernamentales como desde organizaciones no estatales. Uno de estos ámbitos específicos es el de los "menores en riesgo o vulnerables" a los que, de acuerdo con determinados perfiles característicos, hemos denominado "niños que permanecen en las calles".
\end{abstract}

En el trabajo se muestran los resultados de una investigación llevada a cabo con jóvenes entre los 9 y 17 años que pasan numerosas horas en la calle, ejercen diversas actividades o que viven permanentemente en este medio de la ciudad de Lima (Perú). Se analizan el perfil del menor y su contexto, sus percepciones sobre el concepto y beneficios atribuidos a las drogas (inhalantes, alcohol y tabaco) y la relación de éstas con las conductas de consumo, todo ello desde la óptica de la educación social.

Palabras clave: Niños de la calle, niños en la calle, educación social, drogodependencias, percepciones y creencias sociales

\section{SOCIAL EDUCATION AND VULNERABILITY OF CHILDREN WHO STAY ON THE STREET}

\section{Abstract}

Arising from the development of Welfare State in Europe after the Second War World-on western societies, frequently, social attentions and services have extended and changed, in the same way of sceneries and areas from social pedagogy, aim at diverse levels of population inside the field of socioeducational performance. In Iberian Peninsula, specially in Peru, since the academic viewpoint, social pedagogy does not have a meaningful role as in Europe.

However, there is a sociopolitical praxis in different boundaries which are developed such as in governmental institutions as in private organizations. One or these specific scopes is "children in risk or vulnerable children", who since their typical profiles, we have named "children who stay on the streets". Results of a research project are shown

* Doctora en Pedagogía, profesora e investigadora en el Departamento de Pedagogía de la Universidad de Granada (España). Líneas de investigación: diversos escenarios de la Educación Social, especialmente en drogodependencias, menores en riesgo, género y educación intergeneracional. fanntab@ugr.es

Texto recibido el 13-02-2006 y aprobado: 20-04-2006 
in the paper. It was conducted with participants among nine to seventeen years, who stay for hours on the street. They make various activities and live permanently in this setting of Lima. It analyzes the profile of minor and hiss context, his perceptions and some benefits given by drugs (inhalants, alcohol and tobacco). And their relationship to addictive behaviour; everything since the point of view of Social Education.

Key words: Children on the street, homeless children, social education, drug abuse, perceptions and social believe.

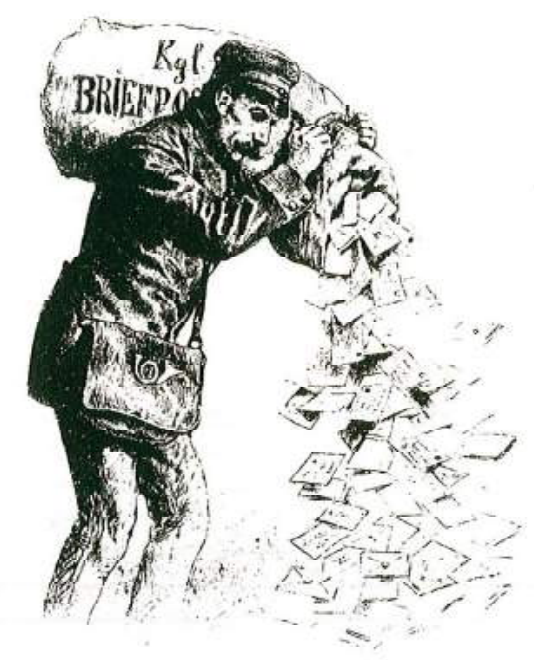

INTRODUCCIÓN

Todas las culturas comparten la visión de que cuanto más joven sea una persona, más vulnerable es física y psicológicamente, y menos capacidad tiene de bastarse por sí misma. Los límites de edad son un reflejo formal del juicio que se forma sobre la evolución de las capacidades y las responsabilidades de los menores; sin embargo, éstos difieren de una actividad a otra y entre los distintos países.

El grupo de los niños y las niñas, según la Convención de los Derechos del Niño de 1989, lo conforman todas las personas menores de 18 años. Sin embargo, dentro de dicha población existe una gran diversidad tanto etaria como psicosomática, socioeconómica, cultural, etc. De ellos distinguimos a una proporción muy significativa que se encuentra en las calles, en especial en contextos de precariedad y pobreza, situados generalmente en las grandes ciudades de los países en vías de desarrollo.
Así observamos que más de mil millones de menores no disfrutan del desarrollo y protección que prometió la Convención sobre el Tratado de Derechos Humanos más ampliamente aprobado en el mundo (Unicef, 2005). La pobreza crea una necesidad económica que puede obligar a los niños y niñas a realizar trabajos que, en la opinión de Kofi A. Annan (2005: viii), deniegan a la infancia su dignidad, pone en peligro sus vidas y limitan su potencial.

En las calles se distinguen (AnderEgg, 1995; Añaños, 2000; Unicef, 1989; Vidal, 2004) a niños y niñas que cuentan con la familia pero se encuentran en ella efectuando distintas actividades ("menores/niños en la calle"), otros que carecen completamente de referentes paternos y para quienes la calle se convierte en su hábitat cotidiano ("menores/niños de la calle"). En este caso, nos ocuparemos de ambos tipos de sujetos a los que hemos denominado "niños que permanecen en las calles".

La calle, para estas personas, se transforma en un espacio donde ejercen diversas actuaciones como el desempeño de trabajos peligrosos o ligeros (Convenio 138, OIT; Unicef, 1996), deambulan sin rumbo ni fin concretos, hasta ejecutar actos transgresores o de mendicidad; así se convierte este ambiente adverso y peligroso en un nicho o caldo de cultivo de situaciones y factores que ponen en grave riesgo la integridad del menor, sometiéndole a circunstancias de desprotección, abusos, maltrato, explotación, conductas adictivas, conductas delictivas, etc. (Añaños, 2000). Prueba de ello es la fragilidad de estas personas para iniciarse en el consumo de drogas dentro de esta subcultura (entendiéndose por ésta a las características peculiares o especiales de un grupo o colectivo que los diferencia dentro del marco cultural común de una sociedad concreta), como forma de "superar o evadir" sus problemas de aceptación y relación social, valoradas activamente por el grupo de iguales (Ordóñez, 1995; Añaños, 2002a).

Por otro lado, la situación de la pedagogía social en América Latina, especialmente en Perú, es escasa desde la perspectiva académica en comparación a lo que ocurre en Europa. No obstante, la problemática social, concretamente de los menores objeto del estudio, demuestra una necesidad de actuar desde la educación social. De hecho existen realidades que demuestran su vigencia desde la práctica en los diversos ámbitos o escenarios de la misma, a través de actuaciones desde la política del Estado hasta la intervención de los distintos sectores/organismos sociales sin ánimo de lucro. Una vez más la realidad va por delante de la academia. También hay que destacar que han surgido iniciativas desde algunos movimientos científico-pedagógicos; por ejemplo, es el caso de la Sociedad Ibérica de Pedagogía Social que, desde el "XVIII Seminario Interuniversitario de Pedagogía Social" celebrado en Sevilla (2003) ha ampliado su ámbito geográfico hacia Iberoamérica, pasando a denominarse Sociedad Iberoamericana de Pedagogía Social (SIPS), y erigiéndose en una puerta abierta en el área que permitirá la vinculación y el enriquecimiento de los profesionales universitarios. 
La finalidad del presente artículo responde a objetivos concretos referidos a conocer las características definitorias del colectivo y del contexto, las percepciones y creencias sobre la identificación del concepto de droga $y$ en los beneficios atribuidos a las sustancias estudiadas, y relacionar las percepciones con las conductas de consumo. Partimos de la premisa de que las percepciones, creencias y bondades otorgadas a las drogas constituyen el sustrato sobre el cual se apoyan las actitudes adictivas, y suponen una percepción normativa de la conducta sobre las toxicomanías (Añaños, 2005). Las sustancias estudiadas son los inhalantes, el alcohol y el tabaco porque son las drogas más consumidas de acuerdo con los últimos estudios realizados en este sector de población (Ferrando, 1992; Ordónez y Mejía, 1993; Opción, 2001; Castro de la Mata y Zavaleta, 2001; Vara Horna, 2002; Devida, 2002).

\section{MARCO TEÓRICO}

\subsection{La educación social y los menores/niños en riesgo o vulnerables}

Sobre la educación social (ES), García Molina (2003:32 y ss.) ofrece una extensa definición en la que destaca los aspectos de justicia social así como su carácter político, ético y práxico, en relación con el doble aspecto de profesión socialeducativa:

La educación social es un derecho de la ciudadanía, que se concreta en el reconocimiento de una profesión de carácter pedagógico articulada en torno a acciones de transmisión (de contenidos conceptuales, habilidades técnicas y formas de trato social) y mediación (con la cultura, el entorno social y los demás sujetos), que un agente de la educación (el educador social) realiza con individuos o grupos (sujetos de la educación) en un marco institucional sancionado para tal fin, y que son generadoras de nuevas plataformas culturales y sociales que posibilitan: la incorporación de los sujetos de la educación a la diversidad de las redes sociales (ello abarcaria desde la socialización de la infancia, en su sentido clásico, hasta la posibilidad de posibles tránsitos de los sujetos por lo social, en sentido amplio) y su promoción cultural y social (como apertura a nuevas adquisiciones culturales que amplien o mejoren las perspectivas laborales, de ocio, de relación y participación social, etc.).

Completa su aportación señalando que su finalidad se centra en

contribuir al desarrollo integral de las personas y de la convivencia social, afrontando necesidades y problemas que surgen en la vida cotidiana; compartiendo objetivos, criterios de actuación y principios metodológicos propios de un trabajo social reflexivo, crítico y constructivo, mediante procesos educativos orientados a la transformación de las circunstancias que limitan la integración social de las personas, procurando una mejora significativa del bienestar colectivo $y$, por extensión, haciendo más creíble la legítima aspiración de todos los ciudadanos a una mayor calidad de vida (Ibid.:80).

Por su parte, Santiago Yubero (1996: 18 y ss.) analiza las aportaciones de distintos autores y señala la coincidencia en recomendar la necesidad de compactar el amplio marco de la educación social en dos ámbitos globales de actuación:

1. La animación sociocultural, entendida como elemento de participación y de comunicación de la comunidad.

2. La educación especializada, que tiene su campo de actuación en el intento de buscar soluciones o paliar ciertos problemas psicosociales que se derivan en conductas inadaptadas y problemas de adaptación.

Dicho esto, debemos dar un paso más y señalar las áreas o ámbitos en los que se concreta la praxis social educativa. Senent (2003:62 y ss.) da cuenta de la evolución de los ámbitos de intervención de la educación social, que en España proceden de dos grandes pilares que la desarrollaron: la educación especializada y la animación sociocultural, que han dado lugar hoy a los siguientes ámbitos: animación sociocultural, inserción sociolaboral, educación socioambiental Gestión y difusión cultural, discapacidad físico-psíquica, menores, marginación, delincuencia y toxicomanías, educación de adultos, tercera edad, cooperación para el desarrollo, y nuevos ámbitos como inmigrantes y refugiados, turismo social y ecológico, mediación (social, familiar), intervención social escolar, acogida y adopción, mujeres (promoción, maltrato).

En la misma línea apunta el profesor Ortega (2002, 2003), al manifiestar la ruptura de fronteras entre la educación escolar-extraescolar y el papel de la educación social frente a la violencia escolar, en los temas transversales, la educación familiar, la atención a los problemas de protección y conflicto en la infancia, la transición a la vida activa... apuntando la incorporación de los profesionales de la educación social en contextos escolares. Según este autor, los ámbitos de la ES son: educación no formal, animación sociocultural y desarrollo comunitario, educación especializada (inadaptados y minusválidos), educación de niños y jóvenes disocializados, acogida y adopción, educación de adultos y mayores, ayuda y asistencia a la familia, acción socioeducativa: drogadicción, delincuencia, exclusión..., educación intercultural, inmigrantes, refugiados, minorías étnicas..., pedagogía del ocio, animación socioeconómica, formación ocupacional, inserción sociolaboral, educación socioambiental, gestión y difusión cultural, nuevos ámbitos (deporte, medios audiovisuales de comunicación, educación de la inteligencia emocional, turismo social y ecológico, mediación (social, familiar), mujeres (promoción, maltrato), intervención social escolar, temas transversales, violencia escolar y transición a la vida activa. Como se 
puede visualizar, en todas las clasificaciones el escenario de los menores es una constante.

Así, la educación social no puede restringirse a un grupo de edad concreto, sino adaptado a las exigencias que presenta cada edad o cada colectivo; no obstante, y por motivos metodológicos y de operatividad, se muestra imprescindible realizar actuaciones socioeducativas para grandes grupos separadamente (Añaños, 2003), en ese caso, los niños en riesgo o vulnerables. El espacio de la educación no está circunscrito sólo a la escuela o a la denominada educación formal, sino a todos los procesos educativos, independientemente del concepto institucional en el que tiene lugar: familia, centros de protección, centros de capacitación, centros recreativos, asociaciones comunitarias, la calle, etc.

En ese sentido, resulta prioritario, en la comprensión de los menores en riesgo o vulnerables, concretamente de los "niños que permanecen en las calles", el entendimiento de sus reacciones ante las propuestas educativas. Máxime si tenemos en cuenta que estas personas se encuentran en circunstancias sociales/familiares/económicas, etc., de carencia o insuficiencia material, moral, atención sanitaria; expuestos a conflictos, enfermedades, intemperie... y que han vivido y se han socializado de forma diferente.

La dimensión educativa constituye un aspecto indispensable en la vida de las personas, reconocido como derecho y necesidad básica del menor (Unicef, 1989), defendida y avalada por diversos marcos legislativos y organismos nacionales e internacionales; por ejemplo, la Organización Internacional del Trabajo patrocina la educación obligatoria, además de proteger a los niños contra el trabajo o condiciones laborales inaceptables, velando porque en dicha ocupación no corran peligro la educación y su desarrollo.

La problemática que aborda en este ámbito la educación social necesita, debido a la complejidad y a su dispersión en cuanto a las áreas de actuación que abarca, una programación rigurosa y profesional, sobre todo considerando la eclosión de profesionales que en los últimos años han intervenido desde los distintos sectores de la población, denominados genéricamente como "educadores sociales". Dichos "educadores" cuentan a veces con una gran experiencia, pero admiten la ausencia de marcos conceptuales y metodológicos de referencia, reflexiones sobre la práctica y conocimientos de planificación y programación fundamentados en la teoria y la praxis.

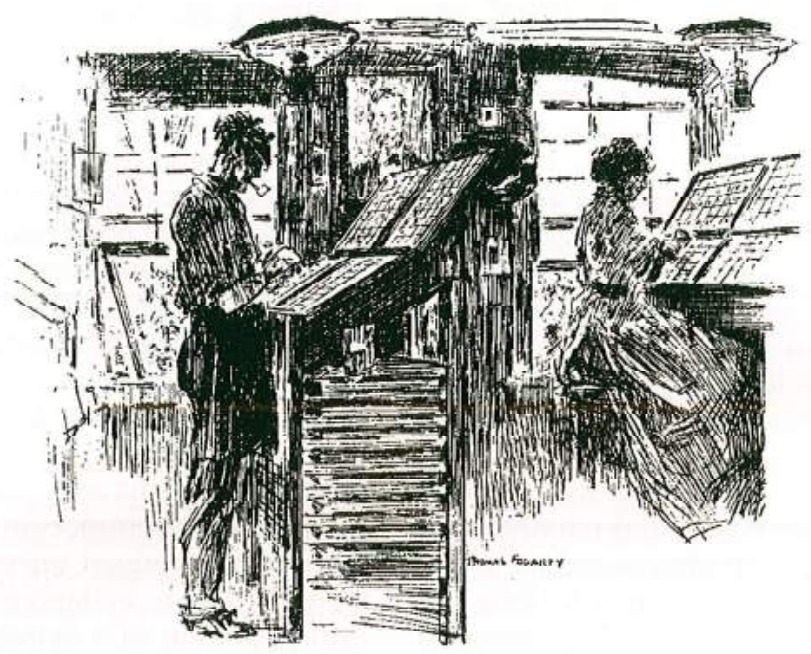

Reforzando la epistemología del educador social, cabe decir que es un profesional distinto al maestro y al trabajador social, pero que educa a través de la convivencia cotidiana. en el marco en el que se desarrolla la vida de los menores que nos preocupan. $Y$ tiene que optar por un modelo de intervención desde lo educativo de forma coherente con la realidad personal y social. Es precisamente ese compromiso educativo el que dará una nueva dimensión al trabajo social, convirtiéndolo en una actividad más involucrada con el cambio social (Petrus, 1997).

\subsection{Los menores/niños vulnerables que permanecen en las calles}

Se entiende por "menor/niño vulnerable" aquellos chicos y chicas en los que confluyen determinadas características personales y sociales que incrementan la probabilidad de implicarse en conductas problemáticas y consumo de drogas (Arbex: 2005:77). El riesgo o la vulnerabilidad se pueden analizar en función de dos grandes referentes:

1. Mayor vulnerabilidad por las situaciones socioculturales en las que están inmersos, es decir, los entornos sociales en los que se están socializando (por ejemplo, barrios o zonas de alto consumo de drogas, delincuencia, desarraigo social, familias multiproblemáticas, conflictos sociales, inmigración con problemas de integración, etc.).

2. Mayor vulnerabilidad por su particular riesgo personal, que vendría determinado por su historia de inadaptación y fracaso escolar, hijos alcohólicos o drogodependientes, niños con experiencias tempranas con las sustancias, etc.

Concretando en datos, más de cien millones de niños sobreviven en las calles de decenas de países, según denuncia de Casa Alianza (2000). Sin la protección de una familia o de una institución, con una opinión 
pública que cierra los ojos y prefiere no verlos en sus barrios, con unos gobiernos insensibles a sus necesidades, estos menores han hecho de la calle su hogar. Un hogar que acoge a los menores en riesgo o vulnerables que hemos denominado "niños que permanecen en las calles" y que incluirían a:

3. "Menores en la calle" (MEC): aquellos que mantienen sus lazos familiares, aunque por lo general inadecuados o deficientes, realizan actividades de subempleo y pasan la mayor parte del tiempo en la calle.

Provienen por lo general de familias de comunidades populares (urbano-marginales) o procedentes de la migración interna de un país. Las familias no logran proporcionarles los satisfactorios básicos y, como consecuencia de una pobreza histórica, estos núcleos no cuentan con herramientas indispensables para la educación y crianza de sus hijos e hijas. Este ambiente adverso plantea cambios radicales en la vida de los menores y con frecuencia se ven obligados a salir de sus hogares, cooperar con la economía familiar, abandonar la escuela o llevar la escolaridad con grandes dificultades, suprimir tiempos de recreación, etc. En este apartado situaríamos a gran parte de los niños y niñas denominados "niños en la calle" o también llamados "niños trabajadores", "niños pobres"...

4. "Menores de la calle" (MDC): aquellos que presentan una situación familiar y social altamente vulnerable, conflictiva o carencial, donde el maltrato, la desintegración o el abandono constituyen una realidad constante (Añaños, 2000)

Según Ander-Egg (1995), los motivos de su permanencia en las calles parecen rondar, por un lado, en un eje central, el factor económico por el cual los menores se ven obligados a salir a trabajar como estrategia de sobrevivencia familiar o para buscar formas de subsistencia por sus propios recursos. Un niño o niña, en última instancia, se queda a vivir en la calle porque escapa del ambiente adverso de su medio, porque es expulsado o porque es abandonado a su suerte. En consecuencia, son sujetos que se encuentran en situación de desamparo/abandono total... la calle es el lugar que se convierte en su hábitat cotidiano... Asimismo, el ambiente reconstituido y la dinámica disfuncional-maltratante de las familias (Opcion, 2001; Ordóñez, y Mejía, 1995) generan el alejamiento total o parcial de los sujetos.

En todos los casos se ven forzados (tácita o explícitamente) a asumir, antes de tiempo, roles de adultos como sobrecompensación, con un destacado costo para el desarrollo psíquico y emocional. Así mismo, los lleva a realizar actividades en condiciones de riesgo físico, psicológico-emocional y moral, donde el consumo de drogas es una de las prácticas que el grupo de iguales de referencia exige o fomenta entre sus integrantes. Todo ello influye en que les disminuyan las posibilidades futuras, y les obliga a desarrollar estrategias de sobrevivencia asociales; no obstante les permite sobrellevar la dura realidad y experiencias dramáticas en que se hallan inmersos (Añaños, 2000, 2002a). Los diversos estudios, centrándonos en el consumo de la población objeto, reflejan resultados muy preocupantes que vislumbran una tendencia ascendente en la ingesta de las drogas, especialmente de inhalantes, alcohol y tabaco (Ferrando, 1992; Ordóñez y Mejía, 1993; Opción, 2001; Vara Horna, 2002).

Todas estas referencias conforman una información muy valiosa que indica la incidencia de estas drogas en la población objeto; por tanto, son razones que justifican que nos decantemos por analizarlas desde la perspectiva de las representaciones sociales.

\subsection{Las percepciones, las creencias y los beneficios atribuidos a las drogas}

Los aspectos sociales, perceptivos, interpretativos, etc., vinculados al uso de las drogas pueden estudiarse recurriendo al concepto de las representaciones sociales. La representación social fue introducida en el mundo científico por Moscovice en 1961, cuando se hace referencia al "pensamiento social". Se trata de buscar estrategias que se manejan para forjar la percepción de las personas, los acontecimientos, el derecho... lo que pondrá en relieve el factor simbólico como predictor y explicativo del comportamiento humano.

La percepción entre los sujetos es distinta, aun cuando la realidad sea idéntica y en ocasiones aparentemente clara, donde la interpretación esté teñida de adscripciones y condicionantes que superen los límites de la objetividad de los individuos, es decir, cada persona elabora su visión particular de lo que tiene delante (familia, barrio, amigos/as, colegio, etc.). El elemento de particularidad conduce al hallazgo, no sólo de una forma de ver, sino a un estilo de ser y hacer, en el que hay una coparticipación grupal o van a recibir una valoración determinada en función de la percepción de los colectivos - extracción de caracteres sociales o culturales- (García, 2005:21).

La imagen de la infancia, según Delgadillo (2004:50-51), se encuentra actualmente entre dos perspectivas: una, que está "desapareciendo" debido a la precocidad en la llegada de la etapa juvenil, relaciones sexuales tempranas, el uso de drogas, etc., y dos, aquélla donde los roles paternos se asumen con mayor compromiso y dedicación, a partir de un reconocimiento del valor de los hijos, de ser sujetos de derechos... extensiva a toda la infancia. Son posturas polarizadas que nos sitúan entre niños y niñas "víctimas", frente a otros muy autónomos, conocedores de los medios, eficientes, etc. Aspectos, 
sin duda, conducentes al análisis y a la construcción cultural permanente del colectivo.

Centrándonos en las drogas, históricamente han sido consideradas con apreciaciones distintas en función de los pueblos y de las culturas. Que el consumo de sustancias haya existido desde siempre es una realidad asumible con facilidad (Escohotado, 1990; Becoña, 1999; Mejía, 2000). La peculiaridad proviene del hecho de que cada momento y cada colectivo le ha atribuido un significado propio con muchas variantes y funciones.

Es incuestionable la importancia dentro de la relación drogas-consumo la presencia del discurso social, entendiéndose por éste la visión, la percepción y sobre todo una evaluación o valoración de algún fenómeno de la realidad (Añaños, 2005:177), en este caso, de los "niños que permanecen en las calles", sobre el consumo de drogas, especialmente inhalantes, alcohol y tabaco.

En definitiva, las prácticas representacionales tienen origen en el laberinto de las condiciones sociales, culturales, económicas, educativas, políticas sobredimensionadas por las disposiciones experienciales del individuo (García, 2005). Del mismo modo, las representaciones sociales -que impregnan el sentido común- ponen en evidencia que el drama social del colectivo está lejos de ser comprendido en su real magnitud y profundidad, y que los estudios realizados, las denuncias reiteradas... y las publicaciones periódicas sobre el tema no han logrado cambiar las condiciones del menor, las actuaciones efectivas en el sector, los lenguajes, las imágenes, las metáforas y las representaciones colectivas sobre éste.

La investigación, teniendo como referencia las representaciones sociales, se dibuja como uno de los escenarios de información e indagación más promisorios en los ámbitos educativos, psicológicos y sociales. Ofrece, entre otras, nuevas explicaciones relacionadas con las dimensiones cognitivas, afectivas y relacionales-sociales.

Develar aspectos representacionales del colectivo de menores en riesgo o vulnerables se convierte en el punto de partida para avanzar en planteamientos concretos y propios de la población objeto del trabajo, especialmente en la prevención $e$ intervención de las drogodependencias. Al mismo tiempo permitirán identificar rasgos comunes, diferenciales y problemáticos del grupo, de tal forma que pueda ser jerarquizado y ponderado en sistemas representacionales que sirvan de referencia y orientación en la formulación de políticas y de intervenciones en el sector. A razón del presente artículo, nos centraremos en la identificación de las sustancias con el concepto de droga y en los beneficios percibidos por su consumo.

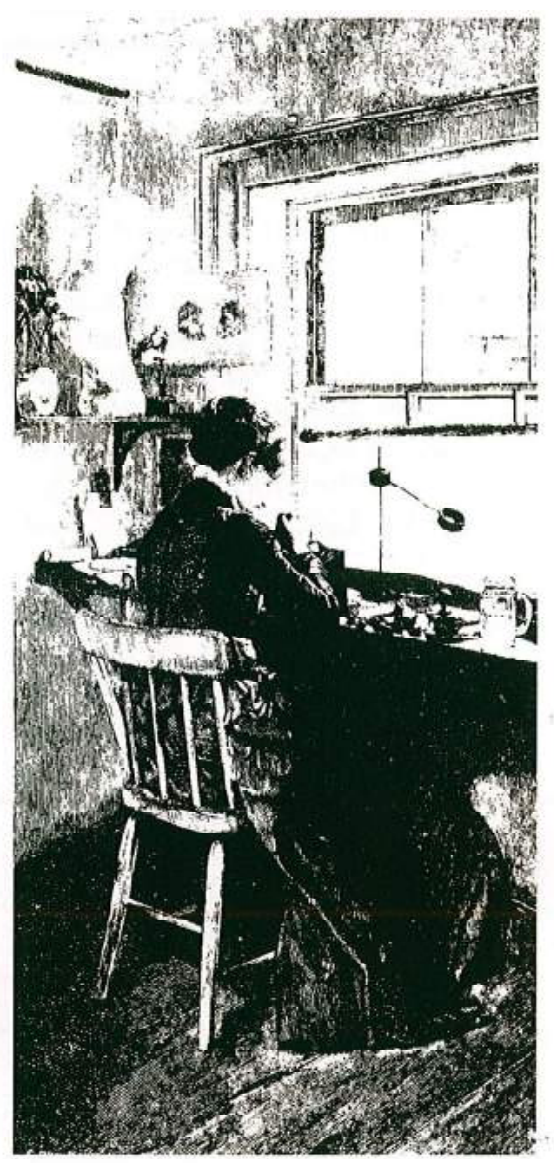

\section{LA INVESTIGACIÓN}

Los "Niños que permanecen en las calles", es el resultado de un estudio descriptivo e interpretativo llevado a cabo en la ciudad de Lima por parte del grupo de investigación HUM 739 de la Universidad de Granada y un grupo de docentes de la Universidad Nacional Mayor de San Marcos (Lima, Perú), que se enfrentan al reto de averiguar, a efectos del presente artículo, las características de los sujetos y su contexto, conocer las percepciones, creencias y beneficios concedidos sobre las drogas de los "niños de la calle" (NDC) y de "niños en la calle" (NEC), en particular las más comunes empleadas en este colectivo: inhalantes, alcohol y tabaco.

La propuesta metodológica de la investigación estuvo dotada de una vía de información con dos vertientes: de un lado el modelo cualitativo, y de otra parte, se cuidó la obtención de informaciones cuantitativas extraídas de la estructura del mismo instrumento (entrevistas en profundidad aleatorias de 28 sujetos). Los métodos de análisis de la información fueron: análisis de contenido y análisis estadístico (SPSS). El periodo de investigación comprendió desde noviembre de 2004 a octubre de 2005.

Los niños y niñas objeto de estudio son sujetos entre 9 y 17 años que desarrollan su vida o pasan numerosas horas de día o de la noche en este espacio -cercado o casco histórico de la ciudad de Lima.

La entrevista personal fue semi-estructurada, en atención a las categorías establecidas en el marco teórico. Recogió de una manera espontánea, en un diálogo abierto y sin limitaciones, las opiniones de los sujetos seleccionados en relación con las drogas que venimos estudiando. La duración aproximada de la entrevista rondó los 30 minutos, fue grabada con la licencia del interesado/a y posteriormente transcrita para su estudio y análisis. 


\subsection{Resultados y discusión}

\subsubsection{Perfil característico del menor y su contexto}

"Los niños que permanecen en las calles" (niños en la calle, NEC, y niños de la calle, NDC), provienen tanto ellos (interior $60,7 \%$, Lima $39,3 \%$ ) como sus progenitores (interior $85,7 \%$, Lima $14,3 \%$ ) fundamentalmente de las distintas provincias del país, y han llegado a la capital como fruto de los movimientos migratorios por razones económicas, sociopolíticas...

Viven con sus familias nucleares 0 parientes en su mayor parte $(67,8 \%)$, pero también el $28,6 \%$ sobrevive solo/a en las calles, y un escaso $3,6 \%$ acude a dormir a centros de acogida. Las personas con quienes comparten sus vivencias (Gráfico 1) son sus padres y hermanos $(46,4 \%)$ o parientes $(17,9 \%)$, amigos/as $(28,6 \%)$, compañeros de centro y solos/as $(3,6 \%)$ respectivamente. E riesgo en las calles es para todos, pero la mayor vulnerabilidad reside en los NDC, así como sentimientos de soledad, de necesidades afectivas-sociales, etc.

La tasa de escolarización, de un poco más de la mitad, registra su vinculación educativa $(57,1 \%)$; no obstante, en los NDC el abandono escolar es de $66,7 \%$. Las explicaciones del absentismo que los menores expresan se refieren a la carencia de recursos económicos, por lo que deben dedicarse a la búsqueda de los mismos $(41,7 \%)$.

Durante la estancia en las calles, desempeñan distintas actividades (gráfico 2), especialmente laborales $(89,3 \%)$, de recreo $(28,6 \%)$ o compartir con sus iguales $(14,3 \%)$. Los tipos de trabajos desarrollados son absolutamente precarios, estacionales, deambulantes... y con multiocupaciones; destacan la venta ambulante (44\%), los servicios de lustrar zapatos (32\%), limpiar coches (16\%), "pesar" a los transeúntes (4\%) y "reciclar" cartones (4\%).

Gráfico 1. Marco de convivencia

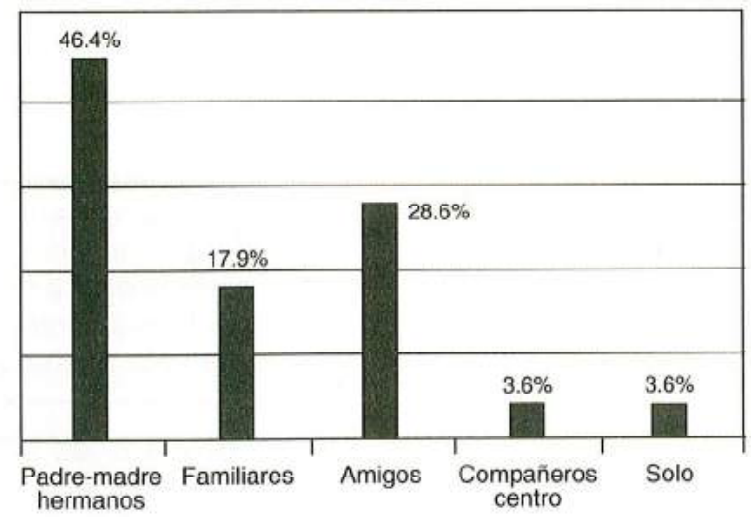

Gráfico 2. ¿Trabajas?

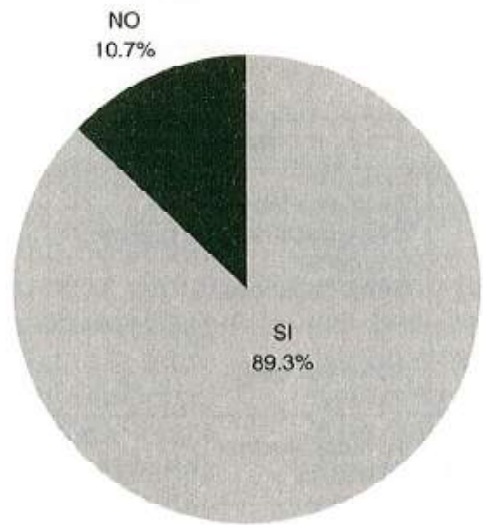

El número de horas en el que se encuentran expuestos en las calles fluctúa entre las 4 y 19 horas, de los cuales el $50 \%$ de los NEC y el $77,8 \%$ de los NDC permanecen más de 11 horas en el medio.

El destino final de sus ingresos se destinan fundamentalmente a la satisfacción de sus necesidades básicas -comida/ropa- $(57,1 \%)$ y a entregar el dinero a su familia $(49,8 \%)$, dato que demuestra que los menores intervienen de forma directa y activa en la economía familiar, siendo ellos mismos quienes se sostienen materialmente. En el caso de los NDC, además de cubrir las cuestiones primarias, también explicitan $(55,6 \%)$ que guardan recursos para adquirir drogas.

\subsubsection{Las percepciones y} creencias sociales sobre las drogas

A razón del artículo, nos centramos en la identificación de las sustancias con el concepto de droga y en los beneficios percibidos por su consumo.

\subsubsection{Identificación de la sustancia como droga}

Las drogas siempre han tenido presencia social y cultural con patrones diferenciados en las distintas épocas y lugares. Los grupos sociales se han relacionado con las sustancias en un equilibrio de coste social y de beneficios individuales y colectivos (apartado 2.1.2.3). Cuando este 
equilibrio se mantiene, la percepción de droga no es evidente, así como la sociedad parece no ser consciente de este fenómeno, es decir, que convive con él (alcohol, tabaco...) y se aprecia como algo natural. En consecuencia, la idea de droga se asocia a toda aquella sustancia "extraña", "ajena", "ilegal", "lejana"... del medio en que nos desenvolvemos, y no se reconocen las sustancias como tales porque se cree que no son droga. Veamos a continuación los datos de la investigación.

Tabla 1. ¿Es droga?

\begin{tabular}{|c|c|c|c|}
\hline & Sí & No & NS/NC \\
\hline Inhalantes & $75 \%$ & $25 \%$ & - \\
\hline Alcohol & $50 \%$ & $46,4 \%$ & $3,6 \%$ \\
\hline Tabaco & $78,6 \%$ & $10,7 \%$ & $10,7 \%$ \\
\hline
\end{tabular}

El reconocimiento de las sustancias como drogas ha sido contundente en el caso del tabaco $(78,6 \%)$ y en los inhalantes (75\%) y en menor medida respecto al alcohol (50\%).

Destacamos las respuestas negativas de la última variable (alcohol, $46,4 \%$ ), que nos indica la baja percepción de droga en el colectivo, así como la gran tolerancia social de que goza la sustancia.

En referencia a los porqués o los razonamientos sobre la identificación o no de la sustancia como droga, vamos a definirlos a través de las siguientes categorías que se extraen de las entrevistas (tablas 2 y 3$)$ :

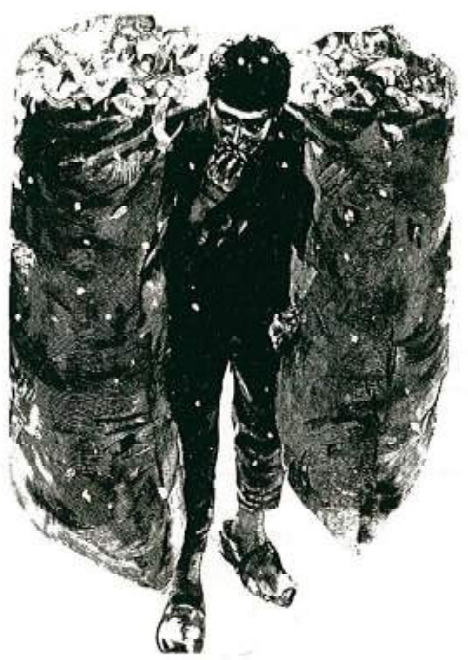

Tabla 2. ¿Por qué es una droga?

\begin{tabular}{|c|c|c|c|}
\hline Categorías & Inhalantes & Alcohol & Tabaco \\
\hline Efectos de las sustancias & $\begin{array}{l}\text { "Te vuelve loco", } \\
\text { "Están decaídos, no pueden } \\
\text { levantarse", } \\
\text { "Duele la cabeza" }\end{array}$ & $\begin{array}{l}\text { "Te emborracha / tontea/cambias/", } \\
\text { "No controlan el cuerpo", } \\
\text { "Te vuelve loco" } \\
\text { "Gritan" }\end{array}$ & "Te malogra la mente" \\
\hline $\begin{array}{l}\text { Carencia de explicación } \\
\text { lógica }\end{array}$ & "No sé por qué" & - & "No sé por qué" \\
\hline Beneficios percibidos & $\begin{array}{l}\text { "Te hace alucinar", } \\
\text { "Te coloca" }\end{array}$ & - & $\begin{array}{l}\text { "Te calienta cuando hace frío" } \\
\text { "Te calma los nervios" }\end{array}$ \\
\hline Afección a la salud & "Hace daño a los pulmones" & "Es malo para la salud" & "Es malo para la salud" \\
\hline Tipo de ingesta & "Sólo se inhala" & - & $\cdot$ \\
\hline Valoraciones negativas & $\begin{array}{l}\text { "Da asco", } \\
\text { "Es malo" }\end{array}$ & - & - \\
\hline $\begin{array}{l}\text { Composición y } \\
\text { características de las } \\
\text { sustancias }\end{array}$ & $\begin{array}{l}\text { "Tiene droga", } \\
\text { "Huele mal, raro, feo" }\end{array}$ & "Contiene algo malo" & $\begin{array}{l}\text { "Tiene nicotina / droga / } \\
\text { alucinógeno/ cocaina" }\end{array}$ \\
\hline Condición social & "La mayoría lo fuma" & - & - \\
\hline $\begin{array}{l}\text { Consecuencias sociales- } \\
\text { personales }\end{array}$ & - & $\begin{array}{l}\text { "Te malogra/malea", } \\
\text { "Te deja tirado en la calle", } \\
\text { "Produce accidentes", } \\
\text { "Te roban en la calle" }\end{array}$ & $\cdot$ \\
\hline Dependencia & $\cdot$ & "Te vuelve vicioso-adicto" & "Te envicia-es adictivo" \\
\hline Cantidad & - & $\begin{array}{l}\text { "Cuando tomas/bebes mucho", } \\
\text { "Se mueren si toman mucho" }\end{array}$ & $\cdot$ \\
\hline
\end{tabular}


Tabla 3. ¿Por qué no es una droga?

\begin{tabular}{|c|c|c|c|}
\hline Categorías & Inhalantes & Alcohol & Tabaco \\
\hline Efectos de las sustancias & - & "Te emborra y nada más" & - \\
\hline $\begin{array}{l}\text { Carencia de explicación } \\
\text { lógica }\end{array}$ & "No sé por qué" & "Porque no" & - \\
\hline Beneficios percibidos & $\begin{array}{l}\text { "Estoy bien", } \\
\text { "Te ayuda a volar / jugar" }\end{array}$ & "Sirve para curar heridas" & - \\
\hline Tipo de ingesta & "Los chicos sólo lo absorben" & "Solo se toma/bebe" & "Solo se fuma" \\
\hline Valoraciones positivas & $\begin{array}{l}\text { "No lo veo malo", } \\
\text { "Es una simple cosa" }\end{array}$ & $\begin{array}{l}\text { "Es normal", } \\
\text { "Se puede controlar el consumo" } \\
\text { "No es malo" }\end{array}$ & - \\
\hline $\begin{array}{l}\text { Composición y } \\
\text { características de las } \\
\text { sustancias }\end{array}$ & "I "Lo sacan de la planta" & $\begin{array}{l}\text { "No tiene elemento químico", } \\
\text { "Tiene origen natural" }\end{array}$ & - \\
\hline Condición social & - & $\begin{array}{l}\text { "Todos toman", } \\
\text { "Si fuera droga, todos seriamos } \\
\text { drogadictos" }\end{array}$ & $\begin{array}{l}\text { "Esto es diferente", } \\
\text { "Todos los consumen", } \\
\text { "Se vende por ahr" }\end{array}$ \\
\hline
\end{tabular}

A partir de las respuestas de los sujetos se organizaron las categorías de las tablas 2 y 3 agrupándose en núcleos temáticos, de las cuales es significativo observar, en gran medida, la contradicción de las variables en cuanto a que se emplean los mismos argumentos tanto por aquellos que reconocen la condición de droga de la sustancia como por aquellos que no la denominan como tal. Destacamos a continuación algunas de gran trascendencia:

1. Efectos de las sustancias: se refieren a la sintomatología que producen las sustancias en el organismo y en el comportamiento $\mathrm{y}$, hasta cierto punto, las consecuencias psicológicas, por ejemplo, cuando expresan que enloquece o afecta la mente. Las respuestas no dan lugar a dudas de que se trata de unos cuadros coherentes con las reacciones de las drogas que se consumen. La falta de reconocimiento de la sustancia como droga, en el caso del alcohol, se da a razón de uno de los efectos, según ellos, pasajeros, de una "simple embriaguez", sin mayores repercusiones y banalizando el consumo.
2. Carencia de explicación lógica: es una presunción de que se trata de una droga, pero no saben exactamente las razones de tal definición. Se refleja en el tabaco y en los inhalantes; llama la atención que en el caso del alcohol no sea así: las explicaciones, entre otras, se deben a que las bebidas alcohólicas no son reconocidas como drogas. Igualmente, esta justificación sirve para expresar lo contrario, constituyendo planteamientos claramente opuestos y que muestran la confusión de los conceptos en el colectivo.

3. Por su composición y características de las sustancias: identificación avalada por composición de los tóxicos. Se observa una confusión en las explicaciones puesto que no se detallan correctamente los componentes activos -situación muy razonable porque gran parte de la población no cuenta con una información exhaustiva ni va a la escuela-, pero se percibe la existencia de algún componente negativo (sólo en el caso del tabaco se reconoce la nicotina); no obstante, se confunden con activos de otras drogas que se consumen de forma parecida a Ios cigarrillos. Este mismo argumento es empleado en sentido contrario (alcohol e inhalantes), es decir, no son drogas porque tienen una procedencia "natural", así como carencia de "químicos". Son datos que ahondan en la contradicción de los pensamientos y del desconocimiento de las drogas.

4. Condición social: es una explicación para justificar el consumo social de forma masiva o tradicional de las sustancias. Centrándonos en el colectivo de los NDC donde la relación adictiva con los inhalantes es mayoritaria, se traduce en: "Algo que muchos consumen no puede ser negativo". En contra, vislumbramos a sujetos que no tipifican las sustancias como tóxicas por estas mismas razones, especialmente en lo relativo al tabaco y al alcohol; el planteamiento es: "Si todos beben, está al alcance libre de las personas y no está mal visto "tomar" o fumar, no es droga". Esta postura refleja la consonancia de unas drogas legales con los códigos culturales vigentes. 
5. Consecuencias sociales-personales: aspecto que hace alusión exclusivamente al colectivo que identifica el alcohol como droga y muestra las consecuencias asociales del consumo y un deterioro comportamental de los sujetos, pero fundamentalmente la exposición grave de riesgo de una persona que ha bebido alcohol al encontrarse en la calle.

6. Dependencia: es sinónimo de adicción, adhesión o hábito que provoca dependencia física o psicológica. Carácter detectado por los sujetos en el alcohol y el tabaco, y definidos como drogas, pero preocupa la baja percepción de esta condición en los inhalantes.

7. Cantidad: es la identificación de la sustancia por la relación cuantitativa que los sujetos consumidores establecen con el tóxico. Así, sólo es tipificado en el caso del alcohol en dos sentidos; por un lado, es droga sólo cuando se bebe mucho y, en contra, en cantidades moderadas incluso "es bueno". Por otro lado, señalan las consecuencias mortales de la excesiva ingesta.

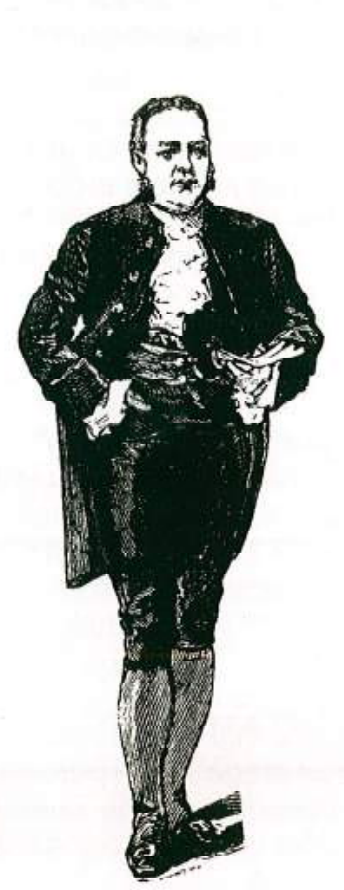

\subsubsection{Beneficios percibidos}

El tándem drogas-beneficios, para Calafat y otros (1985), es el resultado de la relación entre la persona que consume y el producto empleado a través de la necesidad (dependencia física o psíquica) de seguir consumiendo dicha sustancia con clara intención de beneficiarse de sus efectos, tanto a nivel orgánico como sobre el comportamiento (Delgado, Pablos y Sánchez, 1996).

En el presente trabajo, la categorización de la información se efectuó con base en tres grandes ámbitos: las dimensiones psicológica, de la salud y social, y a partir de allí se analizan los significados complacientes, pseudocientíficos, autocomplacientes y

Tabla 4. ¿Qué hace una droga?

\begin{tabular}{|c|c|c|c|}
\hline Beneficios para la salud & $1 \%$ & $A \%$ & $\mathrm{~T} \%$ \\
\hline Evita/disminuye sensación de frío & 21,4 & 10,7 & 35,7 \\
\hline Mitiga/reduce el hambre & 25 & 3,6 & 7,1 \\
\hline Elimina/disminuye el dolor & 7,1 & 14,3 & 3,6 \\
\hline Ayuda a curar enfermedades & 3,6 & - & . \\
\hline Quita/calma la sed & - & 7,1 & - \\
\hline Favorece la digestión & - & - & 3,6 \\
\hline Beneficios psicológicos & $1 \%$ & $\mathbf{A} \%$ & $\mathrm{~T} \%$ \\
\hline Da alegría/estar contento & 14,3 & 35,7 & 10,7 \\
\hline Ayuda a olvidar penas & 14,3 & 7,1 & 3,6 \\
\hline Quita la timidez & 10,7 & 7,1 & 7.1 \\
\hline Contribuye a sentirse más varonil/fem. & 10,7 & 10,7 & 7,1 \\
\hline Facilita descanso-relajación & 21,4 & 7,1 & 21,4 \\
\hline Ofrece sensación de libertad & 3,6 & - & - \\
\hline Ayuda a sentirse ligero & 3,6 & - & - \\
\hline Estimula ver visiones/alucinar & 7,1 & $=$ & - \\
\hline Potencia sentirse seguro/valiente & 14,3 & 10,7 & 14,3 \\
\hline Ayuda a vivir bien y mejor (medio) & 14,3 & 10,7 & 21,4 \\
\hline Contribuye a pensar & 3,6 & - & - \\
\hline Ofrece sentir poder/fortaleza & 3,6 & 3,6 & - \\
\hline Sentirme bien/es bueno & $\cdot$ & - & 17,9 \\
\hline Beneficios sociales & $1 \%$ & $A \%$ & $\mathbf{T} \%$ \\
\hline Ayuda a hacer amigos & 21,4 & 17,9 & 10,7 \\
\hline Facilita el ligue & 7,1 & 10,7 & 33,6 \\
\hline Afianza amistad/confianza & 10.7 & 17.9 & 17.9 \\
\hline Favorece inicio de relaciones sexuales & 3,6 & 7,1 & 7,1 \\
\hline Ayuda a estar acompañado/grupo & 7,1 & 10,7 & 7,1 \\
\hline Estimula compartir/contar problemas & - & 10,7 & - \\
\hline Contribuye a ser más amigable/hablador & - & 3,6 & 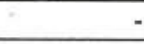 \\
\hline
\end{tabular}

I: inhalante; A: alcohol, T: tabaco. benevolentes de las drogas, si bien en reiteradas ocasiones se producen interacciones entre ellas.

Los beneficios generales más destacados en el total de la muestra en las tres categorías hacen referencia al aspecto festivo y de regocijo del alcohol (35.7\%), de la protección contra el frío del tabaco $(35,7 \%)$ y como elemento de acercamiento ("ligue") de los cigarrillos (33,6\%). Igualmente es destacado el $25 \%$ que registran los inhalantes donde se manifiestan ventajas como la de mitigar las sensaciones de hambre, seguidos del $21,4 \%$ de la misma sustancia: facilita la percepción de relajación/calma/sosiego que produce consumir, reduce la impresión de frío es un dinamizador para hacer más 
amigos/as, respectivamente. En el tabaco, al igual que en los inhalantes, los efectos relajantes están presentes y como una cuestión que ayuda a vivir bien y mejor en las condiciones donde se desenvuelve. Finalmente, el alcohol y el tabaco, con un $17,9 \%$, respectivamente, aluden a sus "propiedades" para relacionarse, entablar nuevas amistades y profundizar dichos lazos.

\subsubsection{Relación con las sustancias}

La aproximación a las sustancias se concreta en el hecho de probar una o más sustancias que ellos consideran son drogas. Así, el 39,3\% dice haber entrado en contacto con algún tóxico, frente al $60,7 \%$ que manifiesta lo contrario. Entrando en más detalle, han consumido: inhalantes (35,7\%), pasta básica de cocaína, PBC (14,3\%), marihuana $(10,7 \%)$, tabaco $(7,1 \%)$, alcohol $(3,6 \%)$ y cocaína $(3,6 \%)$.

Cabe matizar que el consumo real de las drogas lo ha tenido el $71,4 \%$ de los sujetos, distinguiendo dentro de ellos a los NDC. Observamos que el $88,9 \%$ ha entrado en comunión con alguna sustancia; específicamente situando este porcentaje como total, el $100 \%$ probó inhalantes, $44,4 \%$ PBC y marihuana, respectivamente, y $11,1 \%$ alcohol y cocaína.

Centrándonos en las sustancias objeto del estudio (inhalantes, alcohol y tabaco), cuando se les pregunta directamente por el consumo de éstas, las respuestas dan lugar a cuantificar mayores registros que cuando se les pregunta sobre qué drogas han probado. En ese sentido distinguimos (gráfico 3):

Gráfico 3. Contacto con las drogas estudiadas
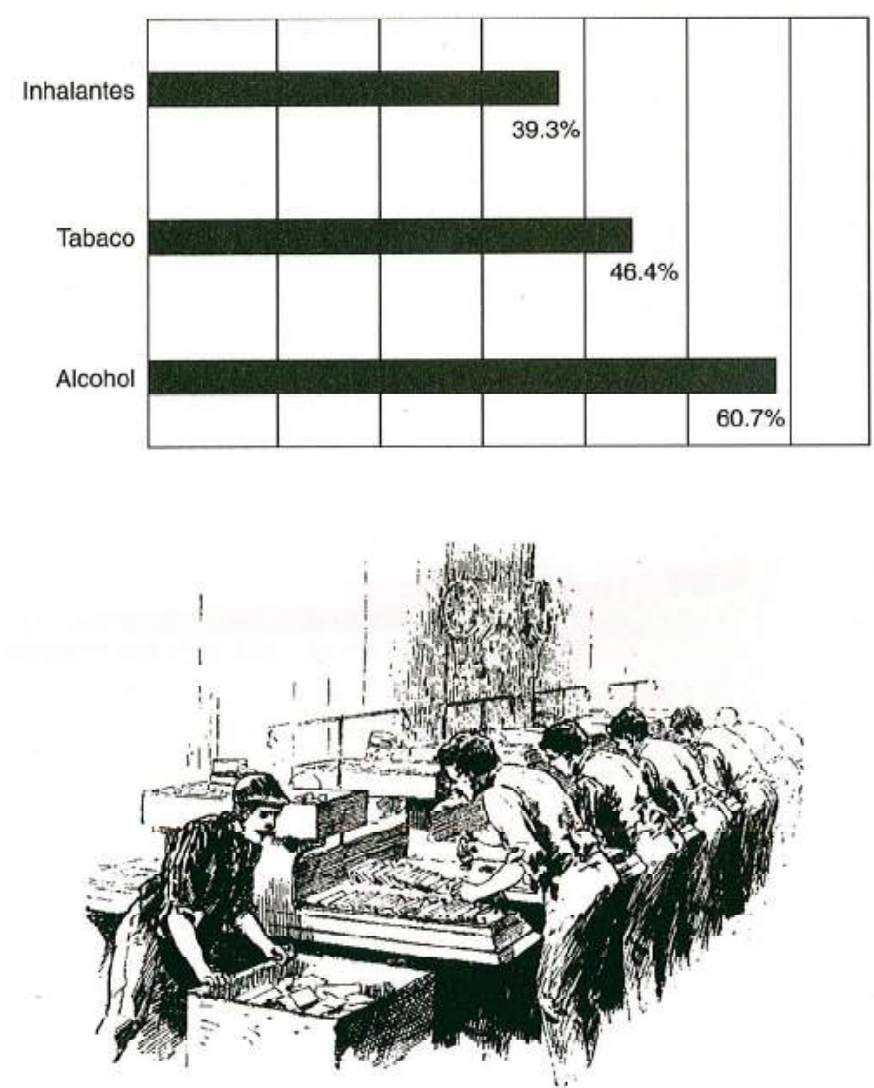

1. Contacto con los inhalantes: la interacción con las distintas sustancias inhalables de la muestra es de $39,3 \%$, de los cuales el $28,6 \%$ son NDC, suponiendo en éstos el $88,9 \%$ del colectivo. Las tipologías más frecuentes son los pegamentos industriales, concretamente, marcas comerciales como "terokal" (72,7\%), "africanito" (45,5\%) y "pegasan" $(9,1 \%)$. No obstante combina o alterna el "terokal" y "africanito" el $27,3 \%$. Otros tipos se refieren al "thiner" $(9,1 \%)$, "gasolina" $(9,1 \%)$ y "keroseno" (9,1\%), producto químico utilizado en los talleres de reparaciones de motos o coches y combustibles obtenidos del petróleo, respectivamente; el policonsumo mezcla "terokal" y "thiner", así como, "gasolina" y "keroseno" $(9,1 \%)$. Los productos inhalables que consumen los sujetos son de fácil y libre obtención, asimismo su costo no es elevado.

2. Contacto con el alcohol: la mayoría de los entrevistados admite haber bebido o seguir ingiriendo alguna sustancia alcóholica $(60,7 \%)$, de los cuales el $25 \%$ son NDC, representando en éstos el $77,8 \%$ del colectivo. Es la sustancia de mayor consumo en el colectivo. Las bebidas más ingeridas son la cerveza con un $41,2 \%$, los licores combinados con refrescos, $29,4 \%$, "pisco puro", 11,8\% (todos NDC), champaña, $11,8 \%$, vino, $5,9 \%$ y cocteles, $5,9 \%$...

3. Contacto con el tabaco: los menores que fuman cigarrillos constituyen el $46,4 \%$ de la muestra, de los cuales el $28,6 \%$ son NDC (suponiendo en ellos el $88,9 \%$ ). Las marcas más consumidas son las Ilamadas "hamilton" y "latino".

Del mismo modo, el alto porcentaje de contacto con las drogas (gráfico 3), pone en relieve una contradicción entre los pensamientos y las conductas, o sea, la mayoría de los menores 
reconoce estas sustancias como drogas (tabla 1), pero la inmensa proporción ha entrado en relación con ellas.

La edad de los sujetos a la hora de tomar contacto/consumo directo con las drogas constituye un hecho de extraordinaria importancia; como veremos, cada vez se produce en edades más prematuras. En el estudio se sitúa en torno a los 10-11 años; específicamente la media de comienzo en el tabaco es la más baja (10,7 años), en el caso del alcohol ligeramente más tarde (11,1 años) y en cuanto a los inhalantes muy cerca al alcohol (11,4 años). Los tramos que se registran más se centran fundamentalmente entre los 10 y 13 años, seguidos próximamente y con puntos semejantes los intervalos de 7 a 9 años y de 14 a 15 años.

Las razones que sirven de motivación para dichos encuentros hacen alusión a las justificaciones que ofrecen éstos, tales como el gozo o gusto de los consumidores al ingerir la sustancia (inhalantes: $21,4 \%$; alcohol: $14,3 \%$ y tabaco: $17,9 \%$ ), la acción imitativa de la conducta adictiva como medio de integración grupal (inhalantes: 10,7\%; alcohol: $7,1 \%$ y tabaco: $10,7 \%$ ), la evasión o intento de desconectar la cruda realidad que les rodea (inhalantes y alcohol: $10,7 \%$, respectivamente) y la asociación festiva del consumo (inhalantes: 7,1\%; alcohol: 10,7\%).

La relación entre las representaciones sociales y la periodicidad de la ingesta depende de varios factores, entre ellos las razones personales y sociales que los "menores que permanecen en las calles" desean obtener, es decir, para conseguir un mismo "bien" o "propiedad" puede relacionarse, de acuerdo con los casos, de modo específico (alcohol, $41,2 \%$, tabaco, 41,2\%; inhalantes, $18,8 \%$ ), ocasional, habitual o compulsivamente (inhalantes $54,4 \%$ y tabaco $23,1 \%$ ). Así, como podemos observar, la gama de efectos positivos es bastante amplia y engloba diversas dimensiones de las personas.
Los espacios y las situaciones responden a unas necesidades simbólicas, la puesta en marcha de rituales y de un imaginario colectivo, en otras palabras, una cultura de la calle, donde las sustancias se encuentran muy presentes y son de fácil acceso y colocan al sujeto en predisposición de consumir. El deseo de estar con los amigos y compartir con ellos las señas de identidad y consolidación del grupo representan las situaciones preferentes para consumir drogas: inhalantes, $17,9 \%$; alcohol, $32,1 \%$; tabaco, 21,4\%. No es desdeñable la permanente disposición a la ingesta de los NDC para inhalar pegamentos $(10,7 \%)$; respectivamente, cuando se encuentran tristes o por sus problemas (alcohol: 10,7\%; inhalantes: $3,1 \%)$, o cuando sienten frío y fuman $(7,1 \%)$, al igual que para sus salidas lúdicas o asistir a fiestas, donde se enfrentan a situaciones propicias para beber alcohol $(17,9 \%)$.

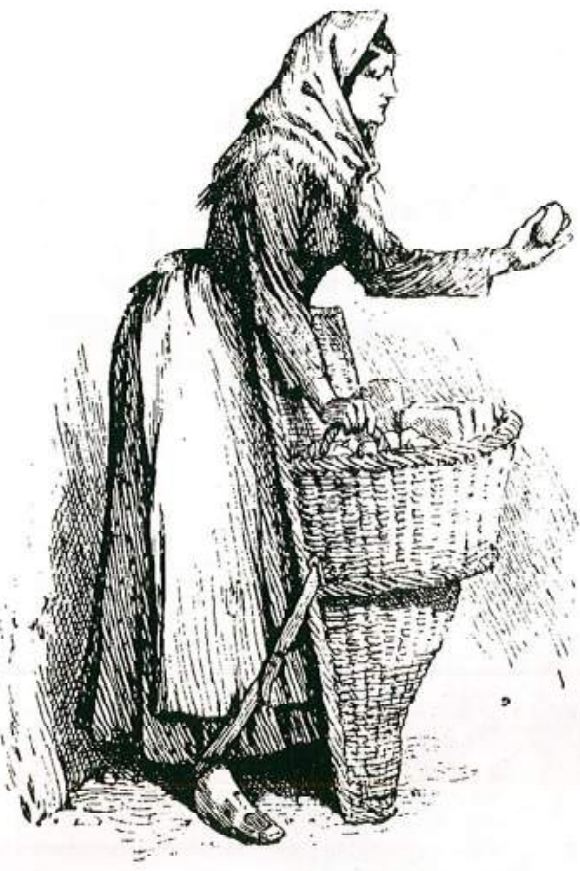
impone claramente la urgencia de contar con políticas integrales que aborden en todos los flancos y dimensiones la problemática actual de la infancia con estas características. En lo que nos atañe, como educadores, es una intervención a través de profesionales especializados en menores en riesgo, especialmente en aquellos que permanecen en las calles, cuya función es de ayuda, formación, orientación y de cooperación al menor que sobrevive en las calles.

El perfil descrito de los "menores que permanecen en la calles" es sumamente preocupante, presentan escaso o carente respaldo material, gran desarraigo, soledad, necesidad imperiosa de buscar afecto, protección y seguridad -especialmente los NDC- , alto indice de absentismo/abandono escolar, explotación laboral durante muchas horas en un medio hostil... donde están presentes distintos riesgos en todos los ámbitos, y las adicciones, sobre todo de los inhalantes, alcohol y tabaco, son la punta de un enorme iceberg.

Las percepciones de los sujetos en cuanto a las sustancias estudiadas se centra en dos aspectos: la identificación del concepto de droga y los beneficios percibidos. La imagen de las drogas es reconocida por la mayoría de los sujetos, aunque de forma confusa, frecuentemente contradictoria y con escasos conocimientos sobre las sustancias. Simbolizan beneficios o consecuencias positivas, a través de distintos efectos que creen sentir al consumir los tóxicos tanto a nivel psicológico, en la salud y en el ámbito social.

Las relaciones drogas-beneficios de los menores se vehiculan mediante la satisfacción de las necesidades primarias, sentimientos de soledad o abandono, relaciones sociales, cubrir la vulnerabilidad, comunicación con los demás, expresar sentimientos y afectos, desarrollar la sexualidad, 
experimentar sensaciones placenteras, etc.; y lo más preocupante es la creencia de que las drogas, en este caso el alcohol, el tabaco o los inhalantes, pueden ayudarles a cumplir el deseo o el gusto.

Los beneficios son los elementos que corroboran las percepciones o valoraciones llenas de benignidad que se otorgan a las drogas: propiedades medicinales, terapéuticas, estéticas, ayudas personales o sociales para estar a gusto, superar los problemas, disfrutar de la vida, minusvalorar los riesgos y las consecuencias sociosanitarias y personales. Sin embargo, todo lo anterior dificulta o limita el desarrollo de sus potencialidades, así como la posibilidad de disfrutar el hecho de ser niño... y beneficiarse de sus derechos.

La comunión de los menores con las sustancias se relaciona con las representaciones sociales, tanto para un contacto inicial como para seguir la vinculación. Así, a pesar de que la mayoría parte de los sujetos reconoce la condición de droga de las sustancias estudiadas, entran en contacto con ellas. Dicho contacto se da cada vez en edades más tempranas.

\section{BIBLIOGRAFÍA}

Ander-EgG, G. Creciendo en situaciones de riesgo. Perfil psico-social de los menores en situación extraordinaria del Estado de Colima. Colima: DIF/Unicef, 1995.

AnNAn, K. "Prólogo". En: Unicef, Estado mundial de la infancia 2005. Nueva York: Unicef, 2005, p. vii.

AÑaños, F. "Los niños/as trabajadores de la calle: un reto para los educadores sociales". En: García Mínguez, J. y otros, Los nuevos yacimientos de empleo y educación social. Granada: Grupo Editorial Universitario, 2002, pp.105-112.

"Algunos entornos generadores de situaciones asociales de riesgo". En: Pedagogía Social n 9, Segunda Época, 2002a, pp. 359-376.
Las motivaciones de la persistencia del consumo se refieren fundamentalmente al disfrute, gusto o satisfactores gratos que se perciben al ingerirlas; en segundo término, las razones de presión, condicionamiento o aliento que ejercen los amigos $y$, en consecuencia, las necesidades de integración influyen poderosamente en el inicio del consumo. Por tanto, el escenario de los amigos se consolida como el marco más importante de consumo, porque la dinámica de ingesta, generalmente, es grupal, siendo dicho entorno la pieza clave de su socialización e identidad. Por consiguiente, las situaciones de ingesta se producen por aliento, condicionamiento, imitación... o estando con ellos.

Por todo lo dicho, expresamos que mediante la concreción de este estudio hemos respondido al desafío de poner en relieve un tema muy delicado; pese a las dificultades, esperamos aportar datos característicos de los sujetos, del contexto, sus hábitos, sus estilos de vida y relaciones, sus percepciones, creencias y beneficios respecto a las drogas estudiadas, con el fin último de poder configurar modelos educativos de transferencia de los símbolos atribuidos a los inhalantes, al alcohol y al tabaco.

Como educadores nos interesa especialmente lo que hay detrás de las ideas, es decir, la simbología que los menores asignan a las drogas, en la medida en que generan dichos modelos e influyen en la conducta. Los significados pueden coincidir o no con las teorías científicas pero siempre es preciso salir a su encuentro, primero para conocer el valor representado y después para interpretar el sentido adjudicado.

Finalmente, nos encontramos ante unos rasgos característicos del menor y de su medio, muy importantes para las políticas de intervención, el marco teórico-científico... y para la actuación del educador social, toda vez que la dinámica de los factores personales y contextuales, particularidades regionales y locales sugieren ser tomadas en cuenta, para adaptar y matizar las consideraciones que modestamente ofrecemos, así como para servir de referente para nuevos hallazgos y resultados que pudieran producir futuras investigaciones en la materia. (coord.). Prevención de las drogas en los jóvenes: ¿Qué prevención? ¿qué drogas?, ¿qué jóvenes? Granada: Grupo Editorial Universitario, 2001.

"El educador social ante las drogodependencias". En: La familia en la sociedad del siglo XXI. Madrid: FAD, 2003, pp.115-122.

(coord.). Las representaciones sociales de los jóvenes sobre las drogas (alcohol, tabaco y cannabis) y su influencia en el consumo. Madrid: Dykinson, 2005.

Arbex, C. (2005). "La vulnerabilidad en los menores: el espacio socioeducativo". En: Pantoja L., Los menores vulnerables y su relación con las drogas. Bilbao:
Instituto Deusto de Drogodependencias, 2005, pp. 77-100.

Calafat, A. y otros. Tú decides. Programa de educación sobre drogas. Palma de Mallorca: Servei d' informació y prevenció de l' abus de drogues, 1985.

Casa Alianza. Vivir en las calles. México: Casa Alianza, 2002.

Castro de la Mata, R. y Zavaleta, M. "Epidemiología de drogas en la población urbana peruana 2001". Monografía de investigación n. ${ }^{9} 20$. Lima: Cedro, 2001.

Chacaltana, J. "Estudio de caso de MANTHOC, un movimiento de niños y niñas trabajadores en Perú". En: Si puedo trabajar... también puedo opinar: diferentes 
enfoques para apoyar a las niños y niñas trabajadores. Lima: Save the Children Suecia, 2000.

Delgado Arcos E., Pablos Márquez, M. y SÁNCHEZ SÁncheZ, D. Programa de prevención de drogodependencias (tabacoy a/cohol) al finalizar la educación primaria. Sevilla: Comisionado para la Droga/Junta de Andalucía, 1996.

Delgadillo, I. "La infancia en la perspectiva de las representaciones sociales". En: Pedagogia y Saberes, n. ${ }^{\circ} 20,2004$. pp. 41-52.

DEVIDA. Encuesta nacional de prevención $y$ consumo de drogas 2002. Lima: Devida, 2002.

EDIs. Los andaluces ante las drogas VIII. Sevilla: Junta de Andalucía, 2003.

FAD. Información general para la prevención de las drogodependencias. Madrid: FAD, 1997.

Ferrando, D. "Uso de sustancias inhalables con fines psicoactivos. Niveles y modalidades en el Perú". En: Psicoactiva n. ${ }^{\circ} 9,1992$.

Garcia Minguez, J. "Las representaciones sociales sobre las drogas". En: Añaños, F. (coord.), Prevención de las drogas en los jóvenes: ¿Qué prevención?, ¿qué drogas?, ¿qué jóvenes?, Granada: Grupo Editorial Universitario, 2001, pp. 45-53.
"Introducción". En: Añaños, F. (coord.), Las representaciones sociales de los jóvenes sobre las drogas (alcohol, tabaco y cannabis) y su influencia en el consumo. Madrid: Dykinson, 2005, pp. 17-38.

García Molina, J. (coord.). De nuevo, la educación social. Madrid: Dykinson, 2003.

Moscoviscı. Psicoanálisis, su imagen y su público. Buenos Aires: Aiqué, 1961.

Oficina Internacional del. Trabajo. A future without child labour: global report under the follow up to the ILO Declaration on Fundamental Principles and Rights at Work. Ginebra: Organización Internacional del Trabajo, 2002.

Opcion. El desafío continúa: "Niños de la calle, sistematización de experiencias del Hogar Juan Miguel'. Lima: Opcion, 2001.

Ordóñez D. "Niños de la calle y consumo de inhalantes: problema multidimensional, intervención terapéutica multiaxial". En: Psicoactiva n. ${ }^{\circ} 13,1995$.

Y MEJIA, M. El trabajo infantil callejero en Lima: Aproximación Descriptiva. Lima: OIT, 1993.

Ortega, J. "Realidades y desafíos". En: Cuadernos de Pedagogían. $³ 21,2003$, pp. 52-54.

(coord.). Nuevos restos de la pedagogía social: la formación del profesorado. Salamanca: Sociedad Ibérica de Pedagogía Social, 2002.
Petrus, A. (coord.). "Concepto de educación social". En Petrus, A. (Coord.), Pedagogía social. Barcelona: Ariel, 1997.

SENENT, J. "Desarrollo contemporáneo de la educación social en Europa. Perspectiva comparada". En: Ruiz C. (coord.), Educación social. Viejos usos y nuevos retos. Valencia: Universidad de Valencia, 2003, pp. 59-90.

UnICEF. Convención sobre los Derechos del Niño. Madrid: Unicef, 1989.

Mejores escuelas: menos trabajo infantil. Santafé de Bogotá, CoIombia: Unicef, 1996.

Estado mundial de la Infancia 1997. Nueva York: Unicef, 1997.

Estado Mundial de la Infancia 2005. Nueva York: Unicef , 2005.

VARa HORna, A. Informe estadístico del primer censo de los niños de la calle. Lima: Asociación por la Defensa de las Minorías, 2002.

VIDAL, C. "Los niños y niñas de la calle". (2004). En: http://www.enbuenasmanos. com/articulos/muestra.asp

Yubero, S. "Educación y sociedad. Temas profesionales en el marco de la educación social". En: Yubero S. y Larrañaga E. (coords.), El desafío de la educación social. Ciudad Real: Servicio de Publicaciones de la Universidad, 1996, pp. 11-22.

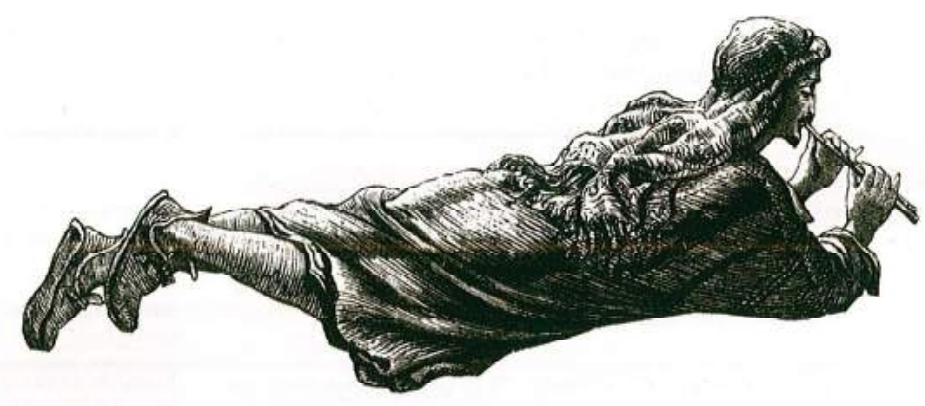

